\title{
Modern CRISPR/Cas9 biotechnology is a current challenge to create elite disease resistant crops cultivars
}

\author{
O.I. Kershanskaya, D.S. Nelidova, G.L. Esenbaeva, G.S. Mukiyanova, S.N. Nelidov
}

Institute Plant Biology and Biotechnology SC, MES RK, Almaty, Kazakhstan

DOI 10.18699/ICG-PlantGen2019-36

(c) Autors, 2019

* e-mail: gen_o.kersh@mail.ru

\begin{abstract}
The technology, a genome-editing tool called CRISPR-Cas9, revolutionized the life sciences when it appeared on the market in 2013. CRISPR (Clustered Regularly Interspaced Short Palindromic Repeats) associated with protein 9 (Cas9) is an RNA-regulated protection mechanism in bacteria and archaea. This is a new "crazy' popular technology of gene editing in humans, animals and higher plants. CRISPR/Cas9 gene-editing is allowing rapid scientific advances in many fields; now it has been shown that crop research can also benefit from this latest exciting technology. A new study of CRISPR-Cas9 technology shows potential to improve crop efficiency.
\end{abstract}

Key words: genome editing; CRISPR/Cas9; breeding perspective; barley.

\section{Introduction}

CRISPR/Cas9 is an RNA-regulated protection mechanism in bacteria and archaea. It is a new popular technology of gene editing in humans, animals and higher plants. The CRISPR/ Cas9 system is a simple, inexpensive and versatile tool for genome editing, resulting in a groundswell of research based on the technique, of which the popularity over the past 6 years has become known as the 'CRISPR craze' (Feng et al., 2013; Brooks et al., 2014; Doudna, Charpentier, 2014; Svitashev et al., 2015; Chandrasekaran et al., 2016; Khlestkina, Shumny, 2016; Gerasimova et al., 2017; Mushtaq et al., 2019). Unlike its predecessors, ZFNs or TALENs, the CRISPR/Cas9 system does not require any protein engineering steps, making it much more straightforward to test multiple gRNAs for each target gene. The CRISPR/Cas9 system consists of a single monomeric protein and a chimeric RNA. A 20-nt sequence in the gRNA confers sequence specificity and cleavage is mediated by the Cas 9 protein. Watson - Crick base pairing with the target DNA sequence is the basis for gRNA-based cleavage. CRISPR is now proving useful as a powerful tool for the improvement of agricultural crops. It is estimated that up to $40 \%$ of harvest is lost worldwide to pests/diseases threatening our food supply. The major crops-wheat, rice, barley-are susceptible to many viral and fungal diseases, which can result in a drastically reduced yield and poor quality grain. Crop cultivars with improved resistance to fungal or viral pathogens will benefit farmers and the local economy by increasing harvest yields and grain quality. The purpose of our research is: elaboration and establishment of a new breakthrough CRISPR/Cas9 biotechnology of genome editing for the creation of elite disease-resistant local barley varieties in Kazakhstan. In this review, some of the first steps in barley CRISPR/Cas9 genome editing for fungal and viral disease resistance are briefly discussed.

\section{Materials and methods}

Plant material: Five local cultivated Kazakhstan barley varieties, differing in yield and resistance to stress. Two of them are brewing barley, and three are feed barley.
Genetic material: target genes, constructs for transformation are: Ac-gene from Amaranthus caudatus, chitinase; CRISPR is a plant construction for barley genome editing by modification or knockout of eIF4E gene, the eukaryotic translation initiation factor that is required by many viruses for multiplication. eIF4E is a plant cellular translation factor essential for the Potyviridae life cycle, and natural point mutations in this gene can confer resistance to potyviruses.

Methods:

I. Gene molecular cloning techniques.

II. Procedure of CRISPR/Cas9 genome editing method:

1. Design sgRNA using the CRISPRdirect website. Requires the identification of target sites with specific sequence criteria, while also avoiding potential off-target effects.

2. Transcribe and screen sgRNA in vitro.

3. Design CRISPR-plant plasmid and constructs for target gene modification.

4. Preparation of Agrobacterium tumefaciens electro-competent cells.

5. Transformation of Agrobacterium tumefaciens competent cells with CRISPR-Cas9 plasmid.

6. Clone confirmation.

7. Deliver sgRNAs and Cas9 to the cell. There are several options for delivering sgRNAs and Cas9 to target cells.

We are developing a method for rapid genotype-independent delivery of genetic material using Agrobacterium pipetting, similar to a pollen-transfection method for other cereals and close to conventional cross-hybridization that requires no tissue culture steps. It is also effective to use bombardment, that is, bioballistic transformation using Bio-Rad' Gen Gun, USA. For S. pyogenes Cas9, the NGG PAM is required immediately downstream of the target site. The two strands of DNA are cut by the HNH and RuvC nuclease domains of Cas9. 8. Detection of Cas9 protein and confirmation of gene editing. Confirm that Cas9 protein is being expressed in target cells.

9. PCR screening. 10. Genotype determination. If there are indels on one or both copies of the target gene, Cas9/ sgRNA-mediated in vitro cleavage reaction can accurately determine the cell's genotype after gene editing. 


\section{Results}

The mechanism of genomic editing using CRISPR/Cas9 includes the next points. For genome editing purposes, generation of a targeted double-strand DNA break (DSB) is the key event that opens up multiple repair options both for the cell and the genome engineer. Such breaks are generally repaired by one of two pathways, homology-directed repair (HDR) or non-homologous end joining (NHEJ). Cells use NHEJ more frequently than HDR because the latter requires a template homologous to the regions flanking the break and to insertions or deletions (INDELs) at its position which can result in functional knock out of a gene.

In all phases of the cell cycle other than S phase, a homologous region of the chromosome is rarely in close enough proximity to act as this template, and thus NHEJ acts as a stop gap to quickly repair the break and maintain chromosomal integrity. NHEJ is an error-prone process that uses ligases, nucleases and polymerases to reseal a break, and generally results in nucleotides being inserted or deleted (indels) in an unpredictable process. If the break occurs in a protein-coding region, these indels will often result in a frame shift mutation and subsequent premature stop codon, abrogating the protein's function.

The magic of CRISPR is in its ability to force a DSB event. Cells must repair DSBs, otherwise there is a risk that cells will die. NHEJ typically leads to a knockout of the targeted genetic element's function. The process of monocots genomic editing includes the following stages: 1 ) selection of target sequences and design of sgRNA; 2) constructing of genetic vectors carrying the nuclease Cas9, sgRNA; 3 ) delivery of the "editing tools" to plant cells; 4) detection of changes in genomic DNA; 5) clean the expression cassette with foreign DNA.

To determine the nucleotide sequence of the target sgRNA, the following web resources in silico were used: CRISPRdirect, CRISPR-PLANT, CRISPR RGEN, etc. The best was CRISPRdirect. The sgRNA sequence for the eIF4E gene in barley (Hordeum vulgare mRNA for eukaryotic translation initiation factor 4E biomaterial PI 39500, Gene Bank accession no. FM244906.2) was selected as AAGGCAGGCCCGCCT. Cas9/sgRNA construct to target the barley eIF4E gene that was expected to disrupt the intact eIF4E protein had been designed. Other sgRNA2 that permit translation of two-thirds of the eIF4E gene protein products will be elaborated soon.

The sgRNA, Cas 9 nuclease, promoters, marker genes were used for constructing genetic vectors. Cassettes of expression of specific sgRNA included the promoter of ubiquitin 6 (U6) from Zea maize L. for sgRNA expression. The start for transcription initiation from the U6 promoter was $\mathrm{G}$ nucleotide. Cassettes for Cas 9 expression consisted of the $35 \mathrm{~S}$ promoter adapted to the Cas 9 coding sequence. The plant selection markers included the hygromycin B resistance gene and the neomycin phosphotransferase gene; the necessary sites of restriction of CRISPR/Cas9 constructs for use in barley is shown in Figure 1, $a$. The size of one sgRNA expression cassette was about $500 \mathrm{~kb}$. The total size of the plasmid was $13300 \mathrm{~kb}$.

As is known, Cas9 has been successfully used to edit the genomes of a number of plants, including barley (Khlestkina, Shumny, 2016; Gerasimova et al., 2017; Mushtaq et al., 2019).
Many genes of Gramineae family plants are characterized by an increased GC-content of the 5' region of the coding part. Cas9, according to the GC-content, corresponding to the structure of the cereal genes $(62.5 \%)$, with a total GC-content of $54.2 \%$, demonstrated a high efficiency of genome editing. In monocot plants, the promoters of ubiquitin genes of maize or rice have been successfully used for Cas9 expression.

Optimally composed expression cassettes made it possible to achieve $90 \%$ of the editing efficiency in T0. Genes of resistance to hygromycin (HPT), under the control of the $35 \mathrm{~S}$ promoter from Cauliflower mosaic virus, are often used as selective markers for editing the genomes of monocotyledonous plants. We have studied knock out and modification of epigenetic factor of virus eIF4E (eukaryotic factor of translation initiation), caused many virus translation initiation in barley. RNAi silencing of eIF $4 E$ has conferred resistance to multiple viruses in melon, broad spectrum resistance to potyviruses in tomato and cucumber (Chandrasekaran et al., 2016). More recently, Arabidopsis complete resistance to Turnip Mosaic Virus has been successfully engineered by editing eIF $4 E$ using the CRISPR/Cas9 tool.

For delivery of editing tools with an expression cassette into barley cells we have developed and patented a method of barley germ-line genetic transformation through pipetting with Agrobacterium tumefaciens, strain EHA105, the expression cassette carrying sgRNA and Cas9 nuclease, to stigma of barley flowers' pistils, exactly before anthesis. Pollen tubes were used as a delivery vector of the targeted construct into mature, but not divided yet, zygote. The method is genotype independent, simple, does not require the tissue culture step, effective at up to $20 \%$ of transformation frequency in the first generation of edited plants.

Confirmation of cloning CRISPR-plant construct and detection of edited events in barley were carried out by PCR, DNA sequencing with using Serial Cloner Program. For PCR screening of monocot CRISPR constructs, we have elaborated the forward primer 5'-GACCAAGCCCGTTATTCTGAC-3' and the reverse primer 5'-AAGTCTGATGCAGCAAGCGAG-3' to amplify the monocot fragment U6-sgRNA(305 bp) to confirm that the selected clones contain U6-sgRNA. The PCR amplification conditions were: $95^{\circ} \mathrm{C}$ for $2 \mathrm{~min}$ for initial denaturation; 30 cycles of $95^{\circ} \mathrm{C} / 30$ seconds, $60^{\circ} \mathrm{C} / 30 \mathrm{sec}$, and $72{ }^{\circ} \mathrm{C} / 30 \mathrm{sec} ; 1$ cycle of $72{ }^{\circ} \mathrm{C} / 5 \mathrm{~min}$.

Using CRISPR/Cas9 editing technology, we have developed $e I F 4 E$ non-transgenic barley mutants that exhibit resistance to three economically important viruses. In Agrobacterium-transformed T0 lines, we found deletions in the $e I F 4 E$ target gene in 8 lines out of 50 (see Figure 1, $b$ ). The same mutations were observed in the $\mathrm{T} 1$ generation, which implies a heterozygous mono-allellic T0 plant, as observed in tomato (Brooks et al., 2014) rice (Zhang et al., 2014) and cucumber (Chandrasekaran et al., 2016). In transgenic lines 4-6, progeny from the $\mathrm{T} 1$ generations showed partial cleavage activity of Cas9.

CRISPR/Cas9 tool editing of eIF4E gene has been successfully engineered in 5 local commercial Kazakhstan barley cultivars, not only in 'Golden Promise' cultivar which is widely popular around the world. 
$a$

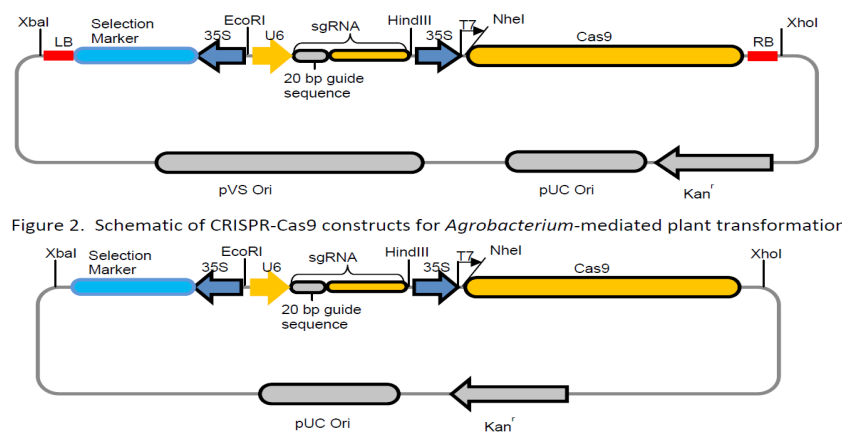

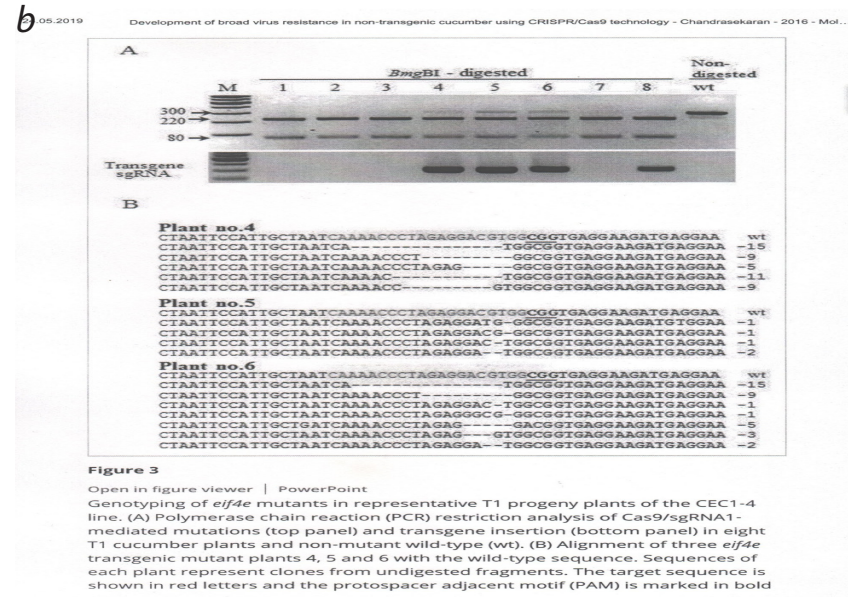

Figure 1. Schematic of the CRISPR-Cas9 construct for Agrobacterium-mediated plant transformation (a), PCR restriction analysis of Cas9/sgRNA elF4E barley mutants (top) and transgene insertion (bottom) in 8 out of 50 edited barley plants and wild-type controls (b).

\section{Discussion}

About 6 years ago the first results on genome editing were achieved on plants, thanks to the use of the relatively simple and convenient CRISPR/Cas9 system, there was a sharp increase in the number of published works reporting successful plant genome editing, including the directed modification of economically valuable genes of cultivated plants: potatoes, cabbage, tomato, maize, rice, wheat, barley, soybeans, sorghum (Feng et al., 2013; Brooks et al., 2014; Svitashev et al., 2015; Khlestkina, Shumny, 2016; Gerasimova et al., 2017). Published works demonstrate the possibility of obtaining nontransgenic plants using the CRISPR/Cas9 system with specific predetermined mutations stably inherited in generations.

The potential efficiency of CRISPR/Cas9 technology is much higher than that of traditional breeding approaches, and excludes the residual portion of the donor genome in editing crops. The average term for the creation of a stable genotype by CRISPR/Cas9 is 2 years, which is a third or a forth as long as the 10-12 years required by backcross methods of conventional breeding or half or a third as long as the time required by modern methods of marker-assisted selection $(\mathrm{MAS})+$ marker double haploid (MDG) + backcross $(\mathrm{BC})$ or obtaining double haploid hybrids. Studies of 145 target genes in 15 crops obtained for 5 years demonstrated the possibility of obtaining modified non-transgenic plants. Editing of 37 genes was accompanied with the improvement of crop yield and stress resistance (Brooks et al., 2014; Khlestkina, Shumny, 2016; Gerasimova et al., 2017). The practical advantage of CRISPR/Cas9 is the ease of multiplexing. The simultaneous introduction of DSBs at multiple sites can be used to edit several genes at once and can be particularly useful to knock out redundant genes or parallel pathways. The same strategy can be used to engineer large genomic deletions or inversions by targeting two widely spaced cleavage sites on the same chromosome (Khlestkina, Shumny, 2016). It is possible to edit several genes simultaneously by introducing multiple or long DNA breaks in the genome to embed a whole complex of useful genes that will be transmitted in the offspring as a single locus. Generally, the CRISPR/Cas9 system for plant genome editing is a breakthrough technology in breeding and a prospect for the creation of elite high yielding crops today and in the nearest future with great social and economic benefit. In the case of using CRISPR/Cas9, several methods are possible for creating of non-transgenic modified plants:

1) by using programmable nucleases, for example, by temporary expression of nuclease components using agroinfiltration or viral vectors, or by direct delivery of components in the form of functional gRNA and Cas9 protein;

2) by integrating the transgenes gRNA and Cas9 protein into a chromosome different from that of the edited gene, so as to get rid of transgenic structures due to independent inheritance in the offspring;

3) transient expression of structures carrying elements of the CRISPR/Cas9 system, without integrating them into genomic DNA. It is assumed that the temporary presence of nucleases and gRNA in the cell may be sufficient to introduce the necessary changes to the genome. It was shown that this principle can work on monocot plants.

Although the European regulatory framework for genetically modified crops focuses on the process and not the product (that is why two identical plants produced by conventional mutagenesis and genetic engineering would be regulated differently under the current guidelines), there is hope and confidence that plants altered by the excision of a few nucleotides using genome editing tools such as CRISPR/Cas9 would not be classified as genetically modified organisms. Removal of undesirable plasmid DNA including Cas9 and guide RNA achieved following segregation and screening of 'clean' plants in the next generation that carry only the edited event. The classification of genome edited plants is currently under review to decide whether new breeding technologies including CRISPR/Cas9 are exempt from GM classification. In the United States, a product-oriented concept has been adopted and it is established that CRISPR/Cas genome edited plants are not GMOs, as they contain no recombinant foreign DNA. Successful results of using CRISPR/Cas 9 technology in agriculture that have been achieved up to this day represent only the very first, initial uses of this exciting technology. Therefore, we can expect many more valuable opportunities for agriculture in the near future. 


\section{Conclusions}

CRISPR/Cas9 technology has revolutionized gene manipulation capabilities in many species including crops. The multiple functions that can be performed with CRISPR/Cas9 and its many derivatives make it a molecular tool that will open new opportunities in a complex world of plant-pathogen interactions and help design durable crop resistance to pathogens.

The CRISPR/Cas9 system for plant genome editing is a prospect for application of breakthrough technologies in breeding.

\section{References}

Doudna J.A., Charpentier E. The new frontier of genome engineering with CRISPR/Cas9. Sci. 2014;346(6213):12580-12596. DOI 10.1126/science. 1258096 .

Feng Z., Zhang B., Ding W., Liu X., Yang D.L., Yang D.L., Wei P., Cao F., Zhu S., Zhang F., Mao Y., Zhu J.K. Efficient genome editing in plants using CRISPR/Cas system. Cell Res. 2013;23:1229-1232. DOI 10.1038/cr.2013.114.

Brooks C., Nekrasov V., Lippman Z.B., Van Eck J. Efficient gene editing in tomato in the first generation using the clustered regularly interspaced short palindromic repeats/CRISPR-associated 9 system. Plant Physiol. 2014;166:1292-1297. DOI 10.1104/pp.114.247577.

Svitashev S., Young J.K., Schwartz C., Gao H., Falco S.C., Cigan A.M. Targeted mutagenesis, precise gene editing, and site-specific gene insertion in maize using Cas9 and guide RNA. Plant Physiol. 2015; 169:931-945. DOI 10.1104/pp.15.00793.

Khlestkina E.K., Shumny V.K. Prospects for application of breakthrough technologies in Breeding: the CRISPR/Cas9 system for plant genome editing. Genetics. 2016;52:774-787. DOI 10.7868/ S0016675816070055.

Chandrasekaran J., Brumin M., Wolf D., Leibman D., Klap C., Pearlsman M., Sherman A., Arazi T., Gal-On A. Development of broad virus resistance in non-transgenic cucumber using CRISPR/Cas9 technology. Mol. Plant Pathol. 2016;17:1140-1153. DOI 10.1111/ mpp. 12375 .

Gerasimova S.V., Khlestkina E.K., Kochetov A.V., Shumny V.K. CRISPR/Cas9 for genome editing and its specialty for monocot plants. Russian J. of Plant Physiol. 2017;64:92-108. DOI 10.1134/ S1021443717010071.

Mushtaq M., Sakina A., Wani S.H., Shikari A.B., Tripathi P., Zaid A., Galla A., Abdelrahman M., Sharma M., Singh A.K., Salgotra R.K. Harnessing genome editing techniques to engineer disease resistance in plants. Front. Plant Sci. 2019. Doi.org/10.3389/fpls.2019. 00550 .

Acknowledgements. The work is supported by the granting program of the Scientific Council of Ministry of Education and Sciences of Republic of Kazakhstan, Project IRN APO5132774, 2018-2020.

Conflict of interest. The authors declare no conflict of interest. 